Case Report

\title{
An Unusual Case of Pancreatic Metastasis from Squamous Cell Carcinoma of the Lung Diagnosed by EUS-Guided Fine Needle Biopsy
}

\author{
Takuya Ishikawa, ${ }^{1,2}$ Yoshiki Hirooka, ${ }^{3}$ Carolin J. Teman, ${ }^{4}$ \\ Hidemi Goto, ${ }^{2}$ and Paul J. Belletrutti ${ }^{1}$ \\ ${ }^{1}$ Division of Gastroenterology and Hepatology, Department of Medicine, University of Calgary, Calgary, AB, Canada \\ ${ }^{2}$ Department of Gastroenterology, Nagoya University Graduate School of Medicine, Nagoya, Japan \\ ${ }^{3}$ Department of Endoscopy, Nagoya University Hospital, Nagoya, Japan \\ ${ }^{4}$ Division of Anatomic Pathology and Cytopathology, Department of Pathology and Laboratory Medicine, \\ University of Calgary, Calgary, AB, Canada
}

Correspondence should be addressed to Paul J. Belletrutti; pjbellet@ucalgary.ca

Received 27 March 2017; Accepted 2 May 2017; Published 17 May 2017

Academic Editor: Vladimir Schraibman

Copyright ( 2017 Takuya Ishikawa et al. This is an open access article distributed under the Creative Commons Attribution License, which permits unrestricted use, distribution, and reproduction in any medium, provided the original work is properly cited.

We report a case of a 70-year-old man who presented with abdominal pain and weight loss, with initial imaging showing simultaneous mass lesions in the pancreas and lungs along with extensive lymphadenopathy in the thorax up to the left supraclavicular region. Core biopsies of the left supraclavicular lymph node showed squamous cell carcinoma, which required differentiation between secondary and primary pancreatic neoplasms. Endoscopic ultrasound-guided sampling using a novel fine needle biopsy system was key to making a definite histological diagnosis and determining the best treatment plan.

\section{Introduction}

Metastatic tumors in the pancreas are uncommon, and most cases are difficult to distinguish from a primary pancreatic cancer. Accurate identification of isolated pancreatic metastases is critical in determining the best surgical and/or medical management [1]. Although lung cancer can metastasize to the pancreas, the frequency ranges according to the histological subtype, and the incidence of pancreas involvement with squamous cell carcinoma is reported as $1.1 \%$ of all pancreatic metastases [2]. Here we report a case of histologically certified metastatic squamous cell carcinoma involving the pancreas from a primary lung cancer, definitively diagnosed with endoscopic ultrasound-guided fine needle biopsy (EUSFNB).

\section{Case Report}

A 70-year-old man presented with a three-month history of progressive abdominal pain and weight loss. His past medical history was unremarkable including no prior history of malignancy. On examination by his primary physician, he was alert and without jaundice or scleral icterus. He had mild epigastric tenderness on abdominal examination, and there were palpable lymph nodes in the left supraclavicular fossa. The remainder of his examination was unremarkable. Laboratory test results were all within normal limits including common blood cell counts, liver chemistries, and serum lipase. Transabdominal ultrasound showed a large distal pancreatic mass. CT scanning revealed a $3.8 \mathrm{~cm}$ hypodense mass in the pancreatic body with lymphadenopathy in the left supraclavicular region. It also showed a $3 \mathrm{~cm}$ lung mass posterior to the left main stem bronchus (Figure 1). Percutaneous biopsy of one of the left supraclavicular lymph nodes revealed squamous cell carcinoma. The patient was referred to our institution for a tissue diagnosis via endoscopic ultrasound (EUS) of the mediastinal mass and to determine the origin of the pancreas mass. Endoscopically there was no abnormality 


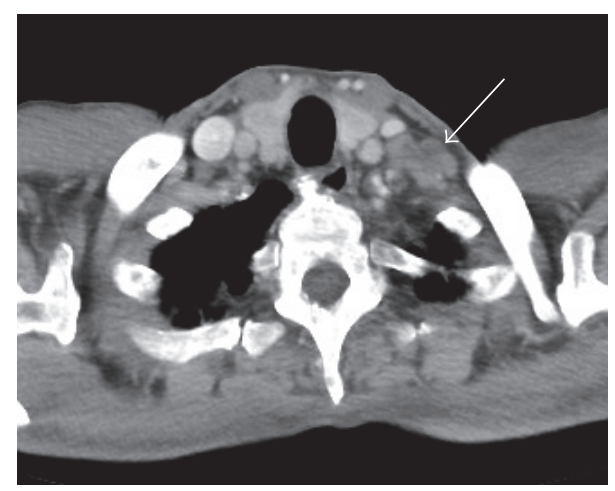

(a)

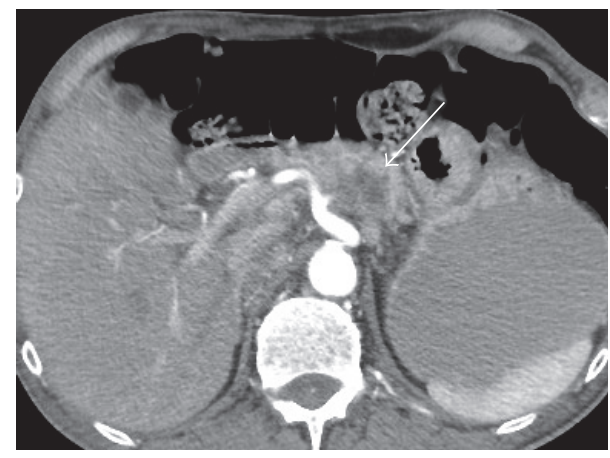

(c)

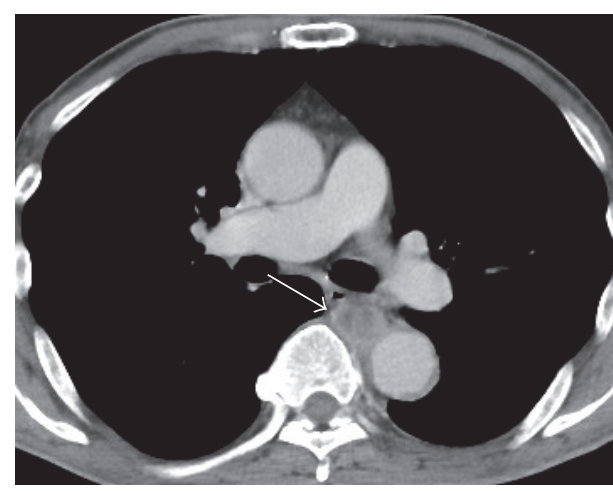

(b)

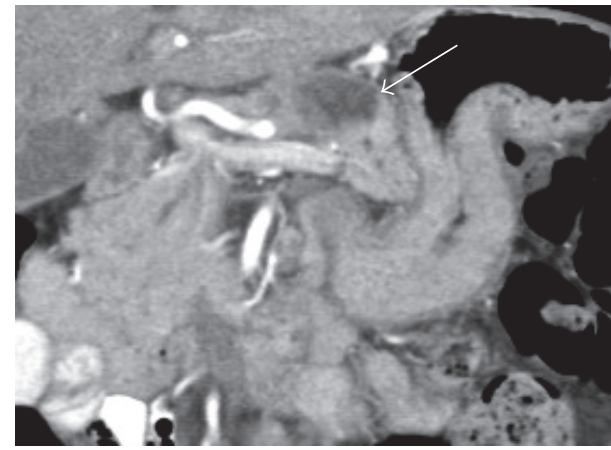

(d)

Figure 1: Computed tomography. (a) A conglomerate of lymph nodes in the left supraclavicular fossa measuring $3 \mathrm{~cm}$ (arrow). (b) A $2 \mathrm{~cm}$ left mid lung mass posterior to the left main stem bronchus (arrow). (c and d) A $3.8 \mathrm{~cm}$ hypodense mass in the pancreatic body with associated ill-defined soft tissue inseparable from the distal celiac axis and its branches (arrow).

in the esophagus or the laryngopharynx. EUS using a lineararray echoendoscope (PENTAX EG-3870UTK) revealed two well-defined hypoechoic lesions with similar echotexture firstly in the mediastinum posterior to the left main stem bronchus and secondly in the pancreatic body. EUS-FNB of these two lesions was performed (Figure 2) with a 25gauge needle using a novel fine needle biopsy system (Beacon SharkCore, Medtronic Corp., Boston, USA). Histopathological examination of both of the specimens revealed a carcinoma morphologically similar to the supraclavicular lymph node biopsy. Immunostains performed on both specimens showed positivity for CK5/6 and p63 (Figures 3 and 4). The patient was thus diagnosed with a metastatic squamous cell carcinoma involving the pancreas from a primary lung cancer. Palliative chemotherapy was planned for the patient.

\section{Discussion}

Clinically apparent pancreatic metastases, while infrequent, are not rare, accounting for up to $3 \%$ of solid pancreatic lesions [3]. Although lung cancer is the second most common primary malignancy that metastasizes to the pancreas (next to renal cell carcinoma), the frequency ranges according to the histological subtype [4], and squamous cell carcinoma is extremely rare. The most frequent type is small cell carcinoma with a pancreatic metastasis incidence of $10 \%$, followed by adenocarcinoma (2.4\%), large cell carcinoma (1.9\%), and finally squamous cell carcinoma with an incidence of only $1.1 \%[2]$.

These metastatic lesions are usually asymptomatic or the symptoms are nonspecific. In many cases, the metastatic lesions are discovered incidentally and are mistaken for primary pancreatic tumors. There are no radiological findings that are pathognomonic of pancreatic metastases. On EUS evaluation, it is reported that metastatic lesions are more likely to have well-defined borders compared with primary pancreatic cancer, but, otherwise, EUS features cannot distinguish between the two groups [5]. Therefore, pathological confirmation of pancreatic tumors is the best method for the diagnosis of pancreatic metastases.

The usefulness of EUS-guided tissue sampling in the diagnosis of pancreatic metastases has previously been reported $[1,5-7]$. However, some of the metastatic tumors to the pancreas (e.g., esophageal and non-SCLC metastases) cannot be definitively confirmed by cytomorphology alone [5], and obtaining optimal histological samples is highly desirable to improve diagnostic accuracy and certainty. Tissue specimens for histological examination can provide the opportunity to immunostain the tissue, further increasing differential diagnostic capabilities for suspected metastatic lesions. Several new "core" FNA needles have been developed, and one such needle, SharkCore, is attracting attention now. 


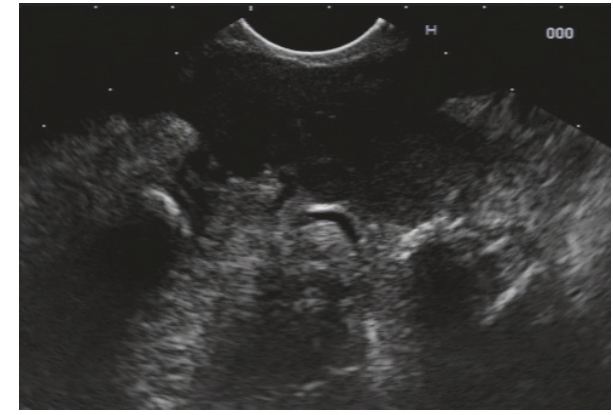

(a)

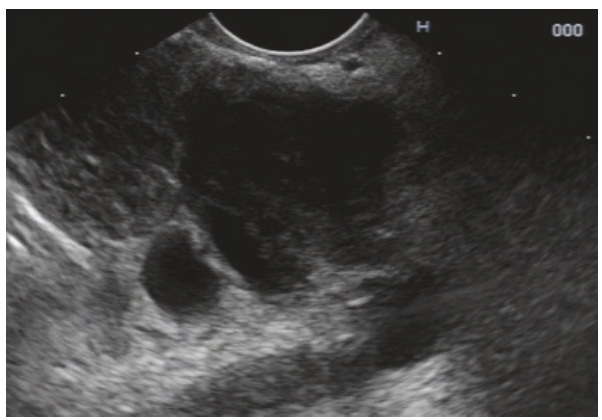

(c)

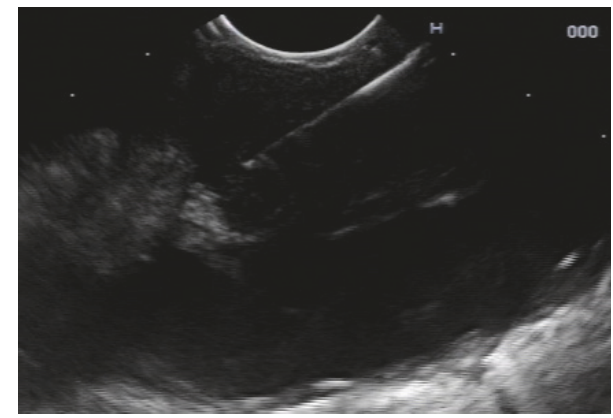

(b)

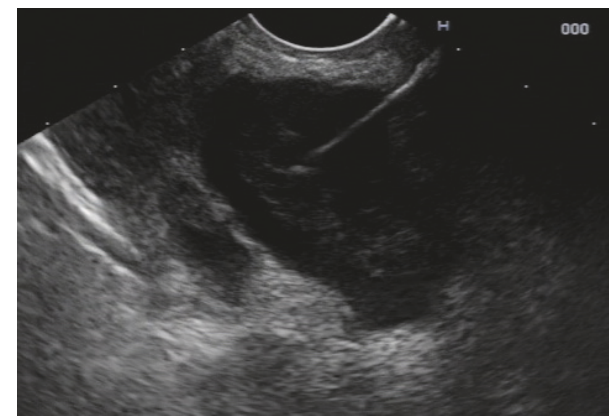

(d)

FIGURE 2: (a) Endoscopic ultrasound (EUS) demonstrating a $3 \mathrm{~cm}$ hypoechoic mass in the left lung. (b) EUS-guided fine needle biopsy (FNB) of the left lung nodule with a 25-gauge needle. (c) EUS demonstrating a $3.8 \mathrm{~cm}$ hypoechoic mass in the pancreatic body abutting the splenic artery. It shows the same internal echotexture as the lesion in the mediastinum. (d) EUS-FNB of the pancreatic mass with a 25-gauge needle.



(a)

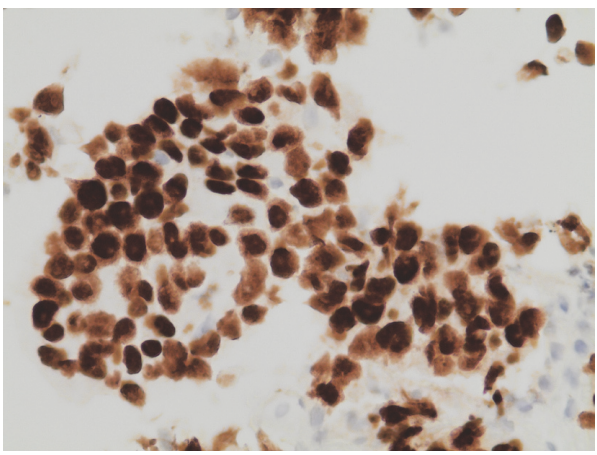

(c)

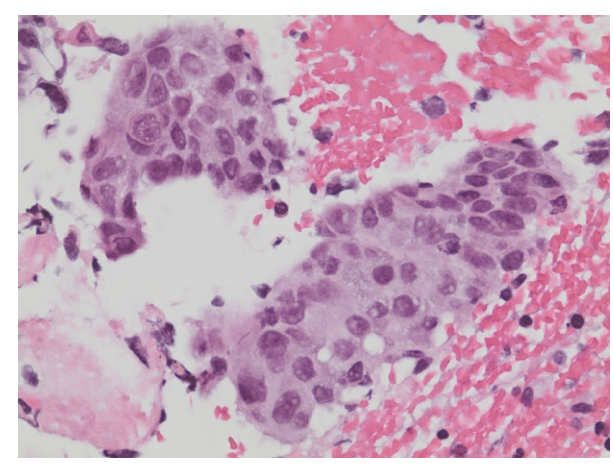

(b)

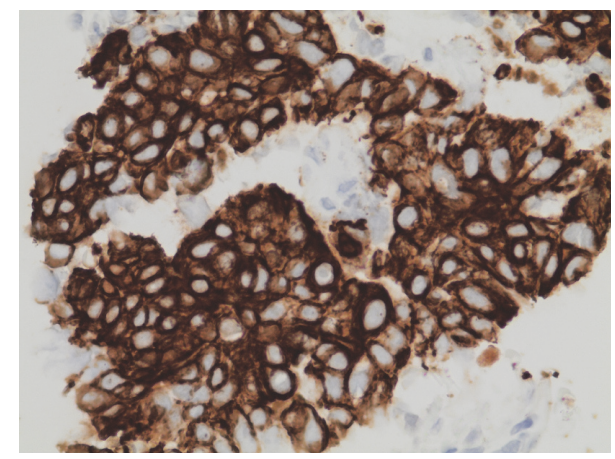

(d)

FIGURE 3: (a) Hematoxylin and eosin staining of a specimen obtained from lung mass with EUS-guided fine needle biopsy. (b) Small core biopsy fragments show invasive carcinoma with clusters and cords of cells that show squamous morphology. (c and d) Immunostains showed that the neoplastic cells express p63 (c) and CK 5/6 (d). 


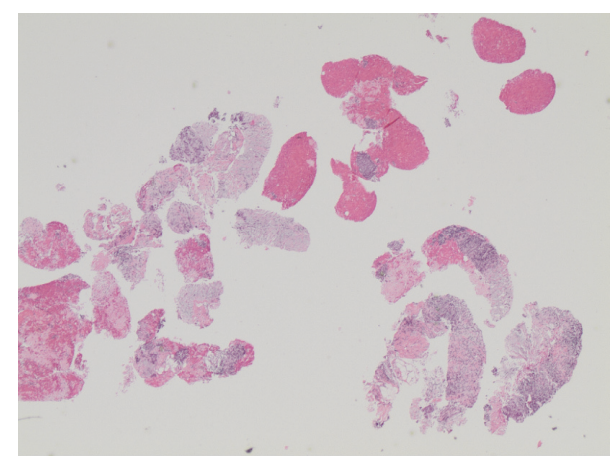

(a)

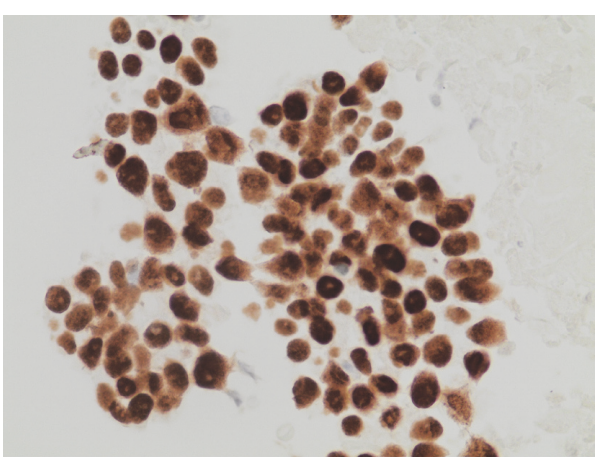

(c)



(b)

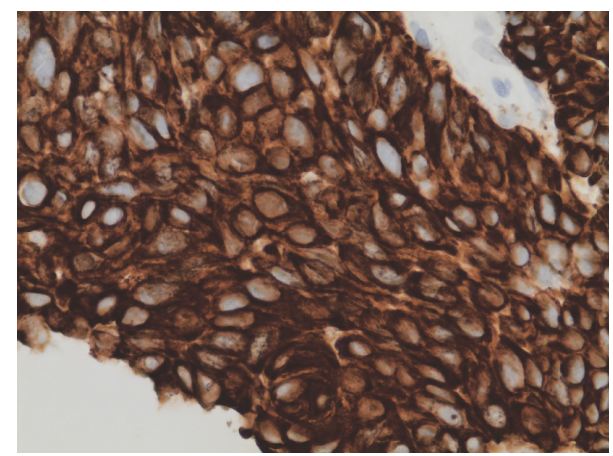

(d)

FIGURE 4: (a) Hematoxylin and eosin staining of a specimen obtained from pancreatic mass with EUS-guided fine needle biopsy. (b) Biopsy fragments show invasive carcinoma morphologically similar to the carcinoma identified in the supraclavicular lymph node and the mediastinal mass. (c and d) Immunostains show that the neoplastic cells express p63 (c) and CK 5/6 (d).

Kandel et al. [8] demonstrated that histological cores were obtained from $95 \%$ of the FNB samples compared with $59 \%$ of the FNA samples in a total of 156 patients, and the histology yield was significantly higher using the SharkCore needle compared with the EUS-FNA needle $(P=0.01)$. Additionally, a large North American multicenter study of the SharkCore needle was recently reported [9]. In this study, a total of 250 lesions were biopsied in 226 patients, and the overall pathologic diagnostic rate was $88 \%$ with a median number of 2 passes. In the present case, the core needle made it possible to obtain adequate histological specimens enabling multiple immunostains to be performed leading to the correct diagnosis and allowing appropriate clinical management to be started without the need for additional time-consuming diagnostic procedures.

In conclusion, although pancreatic metastases from squamous cell carcinoma of the lung are unusual, differentiation from primary pancreatic neoplasms is important. EUS-FNB can allow for a definite histological diagnosis and determination of the most appropriate treatment plan.

\section{Conflicts of Interest}

The authors declare that there are no conflicts of interest regarding the publication of this article.

\section{References}

[1] I. I. El Hajj, J. K. Leblanc, S. Sherman et al., "Endoscopic ultrasound-guided biopsy of pancreatic metastases: a large single-center experience," Pancreas, vol. 42, no. 3, pp. 524-530, 2013.

[2] C. Garcia Vidal, E. Carrillo, and B. Barreiro, "Solitary metastasis to the pancreas in a patient with lung cancer," Archivos de Bronconeumologia, vol. 39, no. 12, Article ID 601, 2003.

[3] J. C. Ardengh, C. V. Lopes, L. F. de Lima et al., "Diagnosis of pancreatic tumors by endoscopic ultrasound-guided fineneedle aspiration," World Journal of Gastroenterology, vol. 13, no. 22, pp. 3112-3116, 2007.

[4] N. Liratzopoulos, E. I. Efremidou, M. S. Papageorgiou, K. Romanidis, G. J. Minopoulos, and K. J. Manolas, "Extrahepatic biliary obstruction due to a solitary pancreatic metastasis of squamous cell lung carcinoma. Case report," Journal of Gastrointestinal and Liver Diseases, vol. 15, no. 1, pp. 73-75, 2006.

[5] J. DeWitt, P. Jowell, J. LeBlanc et al., "EUS-guided FNA of pancreatic metastases: a multicenter experience," Gastrointestinal Endoscopy, vol. 61, no. 6, pp. 689-696, 2005.

[6] M. Sekulic, K. Amin, T. Mettler, L. K. Miller, S. Mallery, and J. R. Stewart, "Pancreatic involvement by metastasizing neoplasms as determined by endoscopic ultrasound-guided fine needle aspiration: a clinicopathologic characterization," Diagnostic Cytopathology, vol. 45, no. 5, pp. 418-425, 2017. 
[7] R. Pannala, K. M. Hallberg-Wallace, A. L. Smith et al., "Endoscopic ultrasound-guided fine needle aspiration cytology of metastatic renal cell carcinoma to the pancreas: a multi-center experience," CytoJournal, vol. 13, no. 1, pp. 13-24, 2016.

[8] P. Kandel, G. Tranesh, A. Nassar et al., "EUS-guided fine needle biopsy sampling using a novel fork-tip needle: a case-control study," Gastrointestinal Endoscopy, vol. 84, no. 6, pp. 1034-1039, 2016.

[9] C. J. DiMaio, J. M. Kolb, P. C. Benias et al., "Initial experience with a novel EUS-guided core biopsy needle (SharkCore): results of a large North American multicenter study," Endoscopy International Open, vol. 4, no. 9, pp. E974-E979, 2016. 


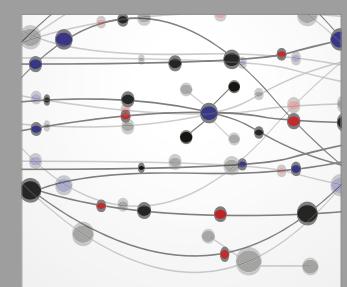

The Scientific World Journal


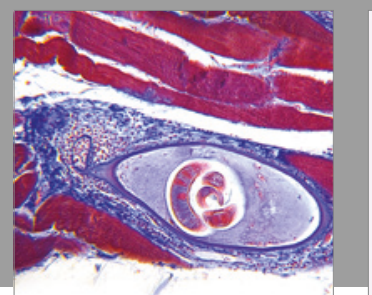

Gastroenterology Research and Practice


\section{Hindawi}

Submit your manuscripts at

https://www.hindawi.com

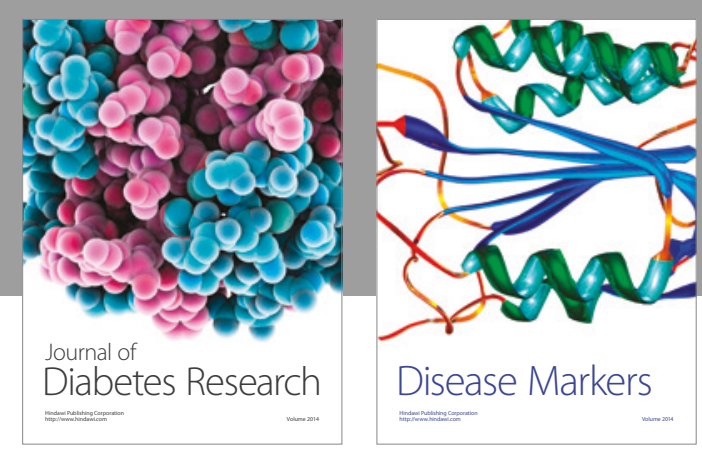

Disease Markers
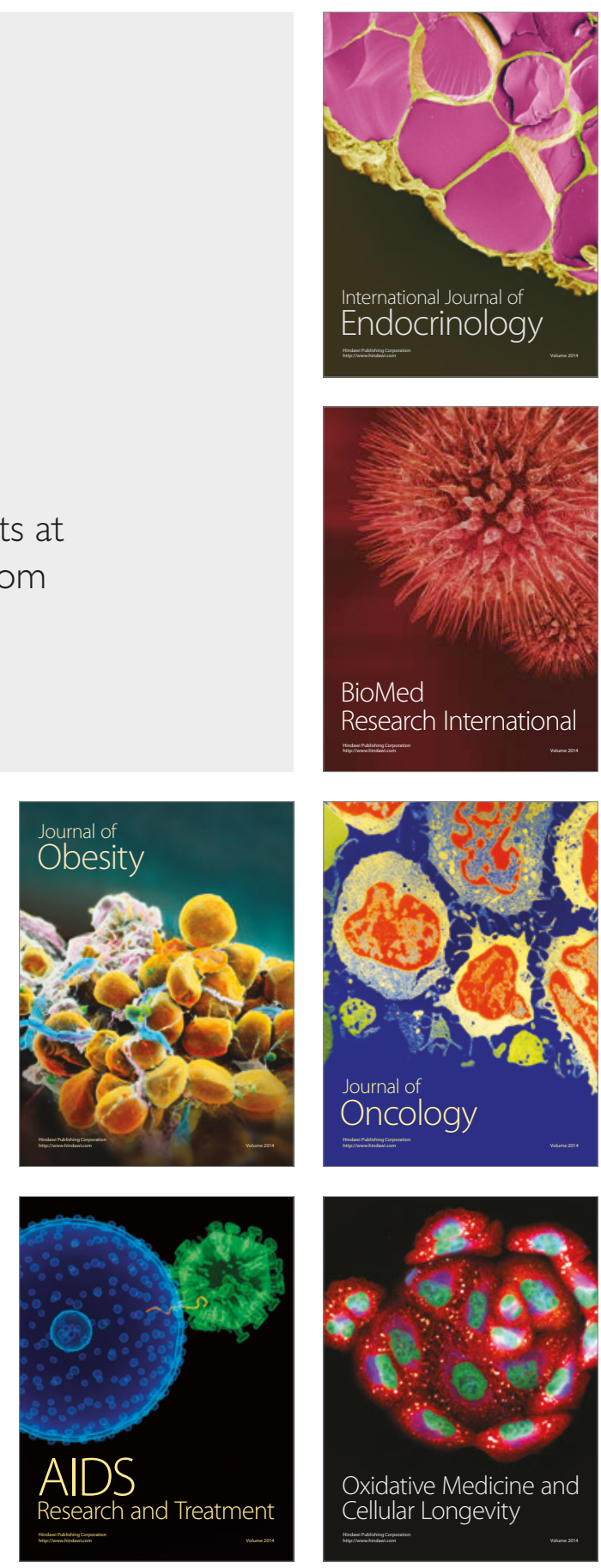